\title{
ACTIVIDAD DE ALGUNOS INSECTICIDAS BIORRACIONALES CONTRA EL GUSANO COGOLLERO
}

\section{ACTIVITY OF SEVERAL BIORATIONAL INSECTICIDES AGAINST THE FALL ARMYWORM}

\author{
María Cristina Zamora ${ }^{1}$, Ana Mabel Martínez ${ }^{1}$, María Soledad Nieto ${ }^{1}$, Marcela Inés Schneider ${ }^{2}$, \\ José Isaac Figueroa ${ }^{1}$ y Samuel Pineda ${ }^{1^{*}}$
}

\begin{abstract}
${ }^{1}$ Instituto de Investigaciones Agropecuarias y Forestales, Universidad Michoacana de San Nicolás de Hidalgo. Km. 95. Carr. Morelia-Zinapécuaro. 58880, Tarímbaro, Michoacán, México. Tel. y Fax: 01 (443) 29583 24. ㄹCentro de Estudios Parasitológicos y de Vectores. Calle 2 N 584. 1900, La Plata, Buenos Aires, Argentina.
\end{abstract}

*Autor para correspondencia (spineda_us@yahoo.com)

\section{RESUMEN}

En este estudio se evaluó el efecto de spinosad, metoxifenocida y azadiractina en huevos y larvas del gusano cogollero, Spodoptera frugiperda (J. E. Smith). La actividad ovicida se determinó en masas de huevos de $<48$ h de edad colocadas sobre papel de estraza, en diferentes concentraciones de cada insecticida diluidos en una solución a $0.01 \%$ de sodio dodecil sulfato. Sólo spinosad y azadiractina mostraron actividad ovicida en concentraciones de hasta $1000 \mathrm{mg} \mathrm{L}^{-1}$ que produjeron un rango de mortalidad entre 12 y $31 \%$. La mortalidad de larvas recién emergidas de los huevos tratados sólo se incrementó $(P \leq \mathbf{0 . 0 5})$ con spinosad y metoxifenocida. En contraste, el peso de las larvas del sexto estadio provenientes de los huevos tratados no fue afectado por los insecticidas, con excepción de spinosad a $3.16 \mathrm{mg}$ i.a. $\mathrm{L}^{-1}$ que incrementó $(\mathbf{P} \leq \mathbf{0 . 0 5})$ el peso de las larvas $(275.5 \mathrm{mg})$ con respecto al testigo $(183.6 \mathrm{mg})$. En un segundo experimento, las larvas del tercer estadio de $S$. frugiperda se trataron por ingestión y tópicamente con los tres compuestos. Los valores de las CL50 de spinosad (2.39 y $0.93 \mathrm{mg}$ i.a. $\mathrm{kg}^{-1}$ de dieta) fueron menores $(P \leq 0.05)$ que los registrados en metoxifenocida $(8.90$ y $4.64 \mathrm{mg}$ i.a. $\mathrm{kg}^{-1}$ de dieta) y azadiractina (14.79 y $63.49 \mathrm{mg}$ i.a. $\mathrm{kg}^{-1}$ de dieta), cuando los insecticidas se mezclaron con la dieta o se aplicaron a la superficie de la misma, respectivamente. En el tratamiento tópico, la DL50 de metoxifenocida (1.27 $\mu$ g i.a. $\mathrm{g}^{-1}$ larva) fue más baja que las de spinosad (2.25 $\mu \mathrm{g}$ i.a. $\mathrm{g}^{-1}$ larva) y azadiractina (7.06 $\mu \mathrm{g}$ i.a. $\mathrm{g}^{-1}$ larva). Los tres insecticidas redujeron significativamente el peso de las larvas de tercer estadio de $S$. frugiperda.

Palabras clave: Spodoptera frugiperda, actividad biológica, azadiractina, metoxifenocida, spinosad.

\section{SUMMARY}

The effect of spinosad, methoxyfenozide, and azadirachtin on eggs and larvae of the fall armyworm Spodoptera frugiperda (J. E. Smith) was evaluated. Ovicidal activity was measured on egg masses of $<48 \mathrm{~h}$ old laid on brown paper, in different concentrations of each insecticide diluted in $0.01 \%$ of sodium dodecyl sulphate. Only spinosad and azadirachtin showed ovicidal activity in concentrations up to $1000 \mathrm{mg} \mathrm{L}^{-1}$, which caused mortalities between 12 and $31 \%$. Mortality of larvae that hatched from treated eggs was increased
$(P \leq 0.05)$ only with spinosad and methoxyfenozide. In contrast, the weigh of sixth instar larvae from treated eggs was not affected by the insecticides, except by spinosad at $3.16 \mathrm{mg}$ a.i. $\mathrm{L}^{-1}$, in this case, the larvae weigh $(275.5 \mathrm{mg})$ became increased $(P \leq 0.05)$ compared to the control $(183.6 \mathrm{mg})$. In another experiment, third instars $S$. frugiperda were treated with the three compounds by ingestion and topical application. The $\mathrm{LC}_{50}$ values of spinosad $(2.39,0.93 \mathrm{mg}$ i.a. $\mathrm{kg}^{-1}$ diet) were lower $(\mathrm{P} \leq \mathbf{0 . 0 5})$ than for methoxyfenozide $(8.90,4.64$ $\mathrm{mg}$ i.a. $\mathrm{kg}^{-1}$ diet) and azadirachtin $\left(14.79,63.49 \mathrm{mg}\right.$ i.a. $\mathrm{kg}^{-1}$ diet), when diet was mixed with insecticides or when were applied on the diet surface, respectively. In topical application, the LDso value of methoxyfenozide (1.27 $\mu \mathrm{g}$ i.a. $\mathrm{g}^{-1}$ larva) was lower than spinosad $\left(2.25 \mu \mathrm{g}\right.$ i.a. $\mathrm{g}^{-1}$ larva) and azadirachtin (7.06 $\mu \mathrm{g}$ i.a. $\mathrm{g}^{-1}$ larva). The three insecticides significantly reduced the larval weight of third instars of $S$. frugiperda.

Index words: Spodoptera frugiperda, biological activity, azadirachtin, methoxyfenozide, spinosad.

\section{INTRODUCCIÓN}

El gusano cogollero [Spodoptera frugiperda (J. E. Smith) (Lepidoptera: Noctuidae)] es la plaga más destructiva del cultivo de maíz (Zea mays L.) en América Latina (Martínez et al., 2004). En los últimos 30 años, el uso intensivo de insecticidas de amplio espectro contra este insecto ha ocasionado el desarrollo de resistencia a la mayoría de los compuestos convencionales registrados para su control, así como contaminación del ambiente (Hruska y Gould, 1997; Morillo y Notz, 2003). Con el fin de disminuir el impacto ambiental derivado de medidas de protección de cultivos, es necesario estudiar nuevas alternativas para el control de $S$. frugiperda, que sean seguras para el ambiente y la salud humana y compatibles con las prácticas de manejo integrado de plagas. Una alternativa promisoria es el uso de agentes biorracionales, como los reguladores del crecimiento de insectos (RCIs) o los 
compuestos derivados de fuentes naturales (Pineda et al., 2006).

Metoxifenocida y azadiractina son dos RCIs de diferente modo de acción. Metoxifenocida pertenece al grupo de los compuestos aceleradores de la muda (CAMs). La estructura química de este compuesto, N'tert-butyl-N'-(3,5-dimetilbenzoil)-3-metoxi-2-metilbenzohidrazida, se basa en la del compuesto prototipo ( $\mathrm{RH}-$ 5849) de este grupo de insecticidas. Esta familia de compuestos mimetiza la función biológica de la hormona 20-hidroxiecdisona e inducen la muda prematura y la muerte debido a la estimulación directa de los receptores ecdisteroidales (Dhadialla et al., 1998). Metoxifenocida es altamente específico contra larvas de lepidópteros por lo que no afecta a mamíferos, peces, pájaros ni a insectos benéficos.

Azadiractina, un tetranortriterpenoide del árbol del nim Azadirachta indica A. Juss (Meliaceae), es uno de los derivados más importantes de plantas usados en el control de insectos plaga (Adel y Sehnal, 2000). Este compuesto tiene propiedades antialimentarias y causa esterilidad en los insectos debido a que altera la concentración de los ecdisteroides y de la hormona juvenil (Schmutterer, 1990).

Spinosad es un biopesticida compuesto por dos lactonas macrocíclicas, Spinosyn A y D, las cuales son metabolitos secundarios producidos durante la fermentación del actinomiceto del suelo Sacharopolyspora spinosa Mertz \& Yao (Thompson et al., 2000). Este compuesto tiene un efecto sinérgico sobre la actividad de la acetilcolina ya que actúa sobre un sitio diferente de los receptores postsinápticos de este neurotransmisor. Spinosad también puede afectar a los receptores del ácido gama aminobutírico (GABA), pero no se sabe con certeza de qué forma lo hace (Watson, 2001). Este insecticida es tóxico por ingestión y contacto y presenta alta actividad en Lepidoptera, Thysanoptera, Isoptera y Diptera (Thompson et al., 2000). Debido a su rápida degradación, baja toxicidad en humanos y bajas dosis de uso de los tres insecticidas descritos, éstos son considerados ambientalmente seguros y constituyen una alternativa a los insecticidas de amplio espectro (Schneider et al., 2004).

El objetivo de este estudio fue determinar los efectos letales y subletales causados por spinosad, metoxifenocida y azadiractina sobre huevo y larva de $S$. frugiperda. Esto con la finalidad de evaluar su toxicidad y su potencial como agentes de control en programas de manejo integrado de plagas.

\section{MATERIALES Y MÉTODOS}

Cría del insecto. Los individuos de $S$. frugiperda se obtuvieron de una colonia mantenida en condiciones controladas de $25 \pm 2{ }^{\circ} \mathrm{C}, 75 \pm 5 \%$ de humedad relativa y un fotoperiodo de 16:8 h (luz:oscuridad). Las larvas se criaron con una dieta artificial elaborada con sémola de maíz, germen de trigo y levadura (adaptada de Poitout y Bues, 1974), y los adultos se alimentaron con una solución de miel de abeja a $15 \%$. Como sustrato de oviposición se utilizó papel de estraza, el cual se reemplazó cada $2 \mathrm{~d}$.

Actividad ovicida y efecto en larvas después de la emergencia. Muestras de 39 a 271 huevos de $<48$ h de edad se colectaron de hembras fertilizadas de $5 \mathrm{~d}$ de edad de $S$. frugiperda. Las tiras de papel de estraza que contenían los huevos se recortaron en círculos pequeños $(\sim 15$ $\mathrm{mm}$ de diámetro), los cuales se sumergieron durante $5 \mathrm{~s}$ en siete concentraciones [(rango de 1 a $1000 \mathrm{mg}$ de ingrediente activo (i.a.) $\left.\mathrm{L}^{-1}\right]$ de spinosad (SpinTor ${ }^{\circledR}$ SC, DowAgrosciences), metoxifenocida (Intrepid ${ }^{\circledR}$ SC, DowAgrosciences) y azadiractina (Aling ${ }^{\circledR}$ EC, Sipcam Inagra). Estas concentraciones se diluyeron en agua destilada más el adherente-dispersante sodio dodecil sulfato (SDS) a $0.01 \%(\mathrm{v}: \mathrm{v})$.

Después de aplicar los tratamientos, los círculos de papel de estraza que contenían los huevos se secaron a temperatura ambiente durante 120 min y se transfirieron a una caja de plástico ventilada de $3 \mathrm{~cm}$ de altura y $9 \mathrm{~cm}$ de diámetro. En cada concentración se incluyó un mínimo de tres y un máximo de seis repeticiones, y cada una consistió de un círculo de papel de estraza con huevos tratados o sin tratar. Las muestras del testigo se sumergieron en agua destilada más SDS. A los $4 \mathrm{~d}$ después del tratamiento se registró el porcentaje de eclosión de huevos en cada concentración de los tres insecticidas. A $1 \mathrm{~d}$ de eclosión se seleccionaron al azar entre 45 y 60 larvas, que se colocaron sobre dieta artificial en un mínimo de tres y un máximo de cuatro cajas de plástico ventiladas (15 larvas por caja). La mortalidad de estas larvas se registró diariamente durante $5 \mathrm{~d}$. También se determinó el peso de las larvas sobrevivientes cuando éstas se encontraban en el sexto estadio (11 d después de su emergencia, en las condiciones de este estudio). Para ello, se seleccionaron al azar y se pesaron individualmente un mínimo de $10 \mathrm{y}$ un máximo de 20 larvas por concentración e insecticida.

Toxicidad larvaria por ingestión. Se utilizaron larvas de tercer estadio (0-8 h después de la ecdisis) de $S$. frugiperda, que se alimentaron continuamente con dieta artificial mezclada o tratada superficialmente con seis concentraciones de spinosad, metoxifenocida o azadiractina. Para 
la incorporación de insecticidas en la dieta, se utilizó un rango de concentraciones de 0.1 a $200 \mathrm{mg}$ de i.a. $\mathrm{kg}^{-1}$ de dieta de cada compuesto, mismas que se prepararon en 20 $\mathrm{mL}$ de agua y se mezclaron con la dieta de cría. Las larvas se colocaron individualmente en celdas de cajas para cultivo de tejidos ( 24 celdas) provistas de un cubo con $8 \mathrm{~g}$ de dieta tratada o sin tratar.

Para aplicar los tratamientos sobre la superficie de dieta, primero se vertieron cerca de $3 \mathrm{~mL}$ de ésta a cada celda de las cajas. Después de la solidificación de la dieta, y con la ayuda de una micropipeta Pipet-Life ${ }^{\circledR}$ (Raining Instrument, LLC, Okaland, CA, EE. UU.), se aplicaron uniformemente $100 \mu \mathrm{L}$ de cada una de las siete concentraciones utilizadas (rango de 0.1 a $316 \mathrm{mg}$ i.a. $\mathrm{L}^{-1}$ ) de cada insecticida, sobre la superficie de la dieta. Después del secado de las soluciones insecticidas $(\sim 2 \mathrm{~h})$, se colocó una larva en cada celda. Para cada concentración e insecticida, y en las dos técnicas de aplicación, se incluyeron cuatro repeticiones de 10 individuos cada una. A las larvas del testigo se les ofreció dieta sin tratar. La mortalidad larvaria se registró diariamente, y las larvas muertas fueron las que no respondían a estímulos aplicados con pinzas entomológicas blandas.

Toxicidad larvaria y reducción de peso por aplicación tópica. Las larvas de tercer estadio de $S$. frugiperda se trataron tópicamente sobre el dorso del tórax con 0.25 $\mu \mathrm{L}$ de spinosad, metoxifenocida o azadiractina. Los insecticidas se disolvieron en acetona y se aplicaron con un microaplicador manual (Burkard, Hertfordshire, Reino Unido). Se utilizaron hasta ocho concentraciones (rango de 1 a $150 \mathrm{mg}$ i.a. $\mathrm{L}^{-1}$; equivalente a las dosis de 0.079 a $11.85 \mu \mathrm{g}$ de i.a. $\mathrm{g}^{-1}$ de larva) de cada insecticida, para tratar 10 larvas por concentración. Se incluyeron cuatro repeticiones y el testigo se trató solamente con $0.25 \mu \mathrm{L}$ de acetona. El peso inicial promedio de las larvas fue $2.52 \pm$ $0.12 \mathrm{mg}$. Después de aplicar los insecticidas, las larvas se colocaron individualmente en celdas de cajas de plástico, como ya se describió, en las cuales se depositó dieta sin tratar como la utilizada en la cría masiva, para asegurar la alimentación de las mismas. La mortalidad de larvas se registró de igual forma que en el tratamiento por ingestión. Además, se determinó la reducción del peso de las larvas sobrevivientes a las $48 \mathrm{~h}$ después del tratamiento, mediante el peso individual de 15 larvas por concentración e insecticida.

Análisis de datos. Los datos del ensayo ovicida (mortalidad en huevos y efectos en larvas provenientes de huevos tratados) y de reducción de peso en larvas del tercer estadio tratadas tópicamente se sometieron a un análisis de varianza. La comparación de medias se hizo con la prueba de diferencia mínima significativa $(\mathrm{P}<0.05)$ con el programa Statgraphics (Graphic software system, STSC Inc., Rockville, MD, EE. UU.). En los casos en los que las varianzas no fueron homogéneas aún con la transformación arcoseno $\sqrt{x}$, se utilizó la prueba no paramétrica de Kruskall-Wallis.

Los datos de mortalidad de los ensayos por ingestión y tópico en larvas de tercer estadio se sometieron a una regresión probit con el programa GLIM (Royal Statistical Society, Inglaterra). Los valores de la concentración letal media (CL50) y dosis letal media (DL50) se calcularon en miligramos de ingrediente activo por kilogramo de dieta y microgramos de ingrediente activo por gramo de larva, respectivamente. Cuando los límites confidenciales (LC) a $95 \%$ no se traslaparon, los valores de las CL50 y DL50 se consideraron significativamente diferentes.

\section{RESULTADOS Y DISCUSIÓN}

Efecto en huevos y en larvas después de la emergencia. Los porcentajes de mortalidad causados por spinosad y azadiractina en huevos de $<48$ h de edad de $S$. frugiperda variaron de 11.6 a $31.3 \%$, que en su mayoría fueron mayores $(\mathrm{P} \leq 0.05)$ al del testigo, que fue $5.3 \%$ (Cuadro 1). Metoxifenocida no causó efecto ovicida significativo, aunque la mayoría de concentraciones triplicó la mortalidad del testigo.

Spinosad fue el insecticida que provocó la mayor mortalidad en larvas que emergieron de huevos tratados (Cuadro 1). Las dos concentraciones más bajas (1 y 3.16 $\mathrm{mg}$ de i.a. $\mathrm{L}^{-1}$ ) provocaron una mortalidad de $67.7 \%$, mientras que las concentraciones más altas provocaron una mortalidad de $100 \%$. En general, la mortalidad de larvas que emergieron de los huevos tratados con metoxifenocida aumentó de forma proporcional al incremento de la concentración (Cuadro 1). Los porcentajes de mortalidad causados por este compuesto (23.3 a $84.4 \%$ ) superaron $(\mathrm{P} \leq 0.05)$ al testigo (9 \%), excepto por la concentración más baja $\left(1 \mathrm{mg}\right.$ de i.a. $\left.\mathrm{L}^{-1}\right)$. Azadiractina causó de 13.3 a $22.2 \%$ de mortalidad en larvas pero tal efecto no fue significativamente superior al testigo.

El peso de larvas del sexto estadio, emergidas de los huevos tratados y que sobrevivieron al efecto de los insecticidas, no fue afectado por los insecticidas, con excepción de spinosad a $3.16 \mathrm{mg}$ i.a. $\mathrm{L}^{-1}$ en donde el peso de las larvas $(275.5 \mathrm{mg})$ se incrementó significativamente con respecto al testigo (183.6 mg) (Cuadro 1). Los valores promedio del peso en los tratamientos con metoxifenocida y azadiractina variaron de 164.1 a 228.9 y 183.8 a $199.5 \mathrm{mg}$, respectivamente. 
Cuadro 1. Efectos de spinosad (SPIN), metoxifenocida (MET) y azadiractina (AZA) en la mortalidad de huevos y en la mortalidad y peso de larvas de Spodoptera frugiperda provenientes de huevos tratados.

\begin{tabular}{|c|c|c|c|}
\hline \multirow{2}{*}{$\begin{array}{l}\text { Concentración } \\
\left(\mathrm{mg} \mathrm{i.a.} \mathrm{L}^{-1}\right)\end{array}$} & \multicolumn{3}{|c|}{ Insecticidas } \\
\hline & SPIN & MET & AZA \\
\hline & \multicolumn{3}{|c|}{ Mortalidad de huevos (\%) } \\
\hline Testigo & $5.3 \pm 2.2 \mathrm{a}$ & & \\
\hline 1 & $18.6 \pm 1.7 \mathrm{bc}$ & $14.8 \pm 1.8 \mathrm{a}$ & $12.5 \pm 3.8 \mathrm{ab}$ \\
\hline 3.16 & $23.6 \pm 7.8 \mathrm{bc}$ & $14.1 \pm 4.3 \mathrm{a}$ & $11.6 \pm 4.5 \mathrm{ab}$ \\
\hline 10 & $21.7 \pm 7.2 \mathrm{bc}$ & $7.8 \pm 1.3 \mathrm{a}$ & $23.5 \pm 2.4 b c$ \\
\hline 31.6 & $11.6 \pm 3.3 \mathrm{ab}$ & $6.3 \pm 1.7 \mathrm{a}$ & $24.4 \pm 2.9 \mathrm{bc}$ \\
\hline 100 & $21.5 \pm 4.1 \mathrm{bc}$ & $7.6 \pm 3.6 \mathrm{a}$ & $31.3 \pm 1.0 \mathrm{c}$ \\
\hline 316 & $21.0 \pm 1.6 \mathrm{bc}$ & $17.5 \pm 3.8 \mathrm{a}$ & $21.5 \pm 3.8 b c$ \\
\hline 1000 & $31.0 \pm 4.3 \mathrm{c}$ & $15.0 \pm 4.6 \mathrm{a}$ & $28.0 \pm 8.8 \mathrm{c}$ \\
\hline \multirow[t]{2}{*}{$\begin{array}{l}\text { Valores de F } \\
\text { (concentraciones) }\end{array}$} & $3.1 *$ & $2.2 \mathrm{~ns}$ & $3.3^{*}$ \\
\hline & \multicolumn{3}{|c|}{ Mortalidad de larvas (\%) } \\
\hline Testigo & $8.9 \pm 4.4 \mathrm{a}$ & & \\
\hline 1 & $66.7 \pm 20.0 \mathrm{~b}$ & $23.3 \pm 3.3 \mathrm{a}$ & $16.7 \pm 1.9 \mathrm{a}$ \\
\hline 3.16 & $66.7 \pm 10.2 b$ & $60.0 \pm 6.7 \mathrm{~b}$ & $16.7 \pm 3.3 \mathrm{a}$ \\
\hline 10 & $100.0 \pm 0.0 \mathrm{c}$ & $56.7 \pm 3.3 \mathrm{~b}$ & $13.4 \pm 7.2 \mathrm{a}$ \\
\hline 31.6 & $100.0 \pm 0.0 \mathrm{c}$ & $73.3 \pm 6.7 \mathrm{bc}$ & $22.2 \pm 2.2 \mathrm{a}$ \\
\hline 100 & $100.0 \pm 0.0 \mathrm{c}$ & $63.3 \pm 10.0 \mathrm{bc}$ & $13.3 \pm 0.0 \mathrm{a}$ \\
\hline 316 & $100.0 \pm 0.0 \mathrm{c}$ & $76.7 \pm 5.8 \mathrm{bc}$ & $13.3 \pm 6.7 \mathrm{a}$ \\
\hline 1000 & $100.0 \pm 0.0 \mathrm{c}$ & $84.4 \pm 8.0 \mathrm{c}$ & $20.0 \pm 6.7 \mathrm{a}$ \\
\hline $\begin{array}{l}\text { Valores de F } \\
\text { (concentraciones) }\end{array}$ & $43.7 * *$ & $16.9 * *$ & $0.7 \mathrm{~ns}$ \\
\hline
\end{tabular}

\begin{tabular}{lccc} 
Testigo & $183.6 \pm 11.9 \mathrm{a}$ & & \\
1 & $145.2 \pm 19.3 \mathrm{a}$ & $228.9 \pm 30.0 \mathrm{a}$ & $199.5 \pm 12.1 \mathrm{a}$ \\
3.16 & $275.5 \pm 26.4 \mathrm{~b}$ & $203.9 \pm 50.9 \mathrm{a}$ & $190.9 \pm 15.1 \mathrm{a}$ \\
10 & $\mathrm{nd}$ & $211.3 \pm 26.8 \mathrm{a}$ & $183.8 \pm 14.3 \mathrm{a}$ \\
31.6 & $\mathrm{nd}$ & $175.8 \pm 28.5 \mathrm{a}$ & $197.3 \pm 21.5 \mathrm{a}$ \\
100 & $\mathrm{nd}$ & $190.8 \pm 33.2 \mathrm{a}$ & $199.4 \pm 18.7 \mathrm{a}$ \\
316 & $\mathrm{nd}$ & $173.4 \pm 7.0 \mathrm{a}$ & $198.4 \pm 15.0 \mathrm{a}$ \\
1000 & $\mathrm{nd}$ & $164.1 \pm 20.7 \mathrm{a}$ & $197.5 \pm 16.0 \mathrm{a}$ \\
Valores de $\mathbf{F}$ & & & \\
(concentraciones) & $11.4^{* *}$ & $5.5 \mathrm{~ns}$ & $5.5 \mathrm{~ns}$ \\
\hline $\begin{array}{l}\text { Medias con letras iguales en cada columna no son estadísticamente dife- } \\
\text { rentes entre sí (DMS, 0.05; y Kruskall-Wallis, 0.05). nd = No determi- }\end{array}$ \\
nado debido a que las larvas murieron inmediatamente después de la \\
emergencia. *, ** Significativo a P $\leq 0.05, \mathrm{P} \leq 0.001$, respectivamente; \\
ns = no significativo.
\end{tabular}

Toxicidad larvaria y reducción de peso. Las larvas de tercer estadio de $S$. frugiperda fueron muy susceptibles a los compuestos que se evaluaron mediante los métodos de ingestión y tópico (Cuadro 2). Spinosad fue más tóxico que metoxifenocida y azadiractina, cuando se mezcló con la dieta de cría o cuando se aplicó a la superficie de la misma (Cuadro 2). Cuando los insecticidas se mezclaron con la dieta, el valor de la CL50 de spinosad fue 3.7 y 6.1 veces menor $(\mathrm{P} \leq 0.05)$ que los registrados para metoxifenocida y azadiractina, respectivamente, con base en los límites confidenciales a $95 \%$. De igual forma, cuando los insecticidas se aplicaron sobre la dieta, el valor de la CL50 de spinosad fue menor $(\mathrm{P} \leq 0.05)$ que los de metoxifenocida y azadiractina.

Cuando los insecticidas se aplicaron tópicamente, los valores respectivos de la DL50 de spinosad y metoxifenocida (2.25 y $1.27 \mu$ g i.a. $\mathrm{g}^{-1}$ larva) no difirieron significativamente, mientras que la DL50 de azadiractina fue $3.1 \mathrm{y}$ 5.5 veces mayor que para spinosad y metoxifenocida, respectivamente.

En general, el tratamiento tópico de spinosad, metoxifenocida y azadiractina redujeron el peso de las larvas del tercer estadio de $S$. frugiperda de forma inversamente proporcional al incremento de la concentración o dosis (Cuadro 3). En spinosad, con excepción de la concentración de $5 \mathrm{mg}$ i.a. $\mathrm{L}^{-1}$, y metoxifenocida, el peso promedio de larvas tratadas varió de 2.3 a 17.6 y de 3.5 a $15.6 \mathrm{mg}$, respectivamente, lo cual representa una reducción significativa del peso entre 27.9 a 90.5 y 35.9 a $85.5 \%$, comparado con las larvas del testigo $(24.4 \mathrm{mg})$. La reducción de peso causado por azadiractina fue similar al registrado en spinosad.

Cuadro 2. Mortalidad de larvas de tercer estadio de Spodoptera frugiperda después de la aplicación de tres pesticidas biorracionales a través de diferentes métodos.

\begin{tabular}{|c|c|c|c|c|c|c|c|c|c|}
\hline Insecticida & Bioensayo & $\begin{array}{c}\text { Tiempo }^{+} \\
\text {(h) }\end{array}$ & $\mathrm{n}^{+}$ & $\mathrm{b} \pm \mathrm{ee}$ & $\mathrm{a}$ & CL50, & DL50 (95\% LC) & $\chi^{2}$ & $\mathrm{gl}$ \\
\hline Metoxifenocida & mezclado en la & 96 & 5 & $0.36 \pm 0.09$ & -0.00013 & 8.90 & $(3.98-34.02)$ & 1.63 & 3 \\
\hline Azadiractina & dieta $H$ & 96 & 6 & $0.56 \pm 0.08$ & 0.0001 & 14.79 & $(8.44-24.68)$ & 1.07 & 4 \\
\hline Spinosad & Insecticida en la & 120 & 3 & $4.54 \pm 0.77$ & -3.00 & 0.93 & $(0.72-1.24)$ & 9.39 & 1 \\
\hline Metoxifenocida & superficie de la & 120 & 6 & $2.84 \pm 0.47$ & -0.002 & 4.64 & $(5.30-6.55)$ & 1.02 & 4 \\
\hline Azadiractina & dieta $H$ & 120 & 4 & $1.52 \pm 0.22$ & 0.002 & 63.49 & $(47.65-84.94)$ & 0.16 & 2 \\
\hline Metoxifenocida & Tópico & 96 & 6 & $0.60 \pm 0.09$ & -0.0003 & 1.27 & $(0.76-2.05)$ & 4.19 & 4 \\
\hline Azadiractina & & 96 & 8 & $0.60 \pm 0.08$ & 0.50 & 7.06 & $(4.67-11.49)$ & 11.31 & 6 \\
\hline
\end{tabular}


Cuadro 3. Promedios y errores estándar de la reducción del peso causada por spinosad, metoxifenocida y azadiractina en larvas de tercer estadio de S. frugiperda tratadas tópicamente $(\mathrm{n}=15$ larvas).

\begin{tabular}{|c|c|c|c|c|}
\hline \multirow{2}{*}{$\begin{array}{l}\text { Concentración } \\
\left(\mathrm{mg} \text { i.a. } \mathrm{L}^{-1}\right)\end{array}$} & \multirow{2}{*}{$\begin{array}{c}\text { Dosis } \\
\left(\mu \mathrm{g} \text { i.a. } \mathrm{g}^{-1} \text { larva }\right)\end{array}$} & \multicolumn{3}{|c|}{ Peso (mg) } \\
\hline & & Spinosad & Metoxifenocida & Azadiractina \\
\hline Testigo & 0 & $24.40 \pm 1.7 \mathrm{a}$ & & \\
\hline 1 & 0.08 & $17.6 \pm 0.6 \mathrm{~b}$ & $15.6 \pm 0.7 b$ & $17.5 \pm 0.6 b$ \\
\hline 5 & 0.39 & $30.0 \pm 0.4 \mathrm{a}$ & $14.1 \pm 0.4 \mathrm{~b}$ & $17.4 \pm 0.2 \mathrm{~b}$ \\
\hline 10 & 0.79 & $17.6 \pm 0.2 b$ & $12.7 \pm 0.2 \mathrm{bc}$ & $14.1 \pm 0.4 \mathrm{c}$ \\
\hline 50 & 3.95 & $14.4 \pm 0.3 \mathrm{c}$ & $6.2 \pm 0.3 \mathrm{~d}$ & $13.7 \pm 0.3 \mathrm{c}$ \\
\hline 100 & 7.90 & $2.5 \pm 0.1 \mathrm{~d}$ & $6.5 \pm 0.3 \mathrm{~d}$ & $2.5 \pm 0.1 \mathrm{~d}$ \\
\hline 150 & 11.85 & $2.3 \pm 0.1 \mathrm{~d}$ & $3.5 \pm 0.2 \mathrm{e}$ & $2.3 \pm 0.1 \mathrm{~d}$ \\
\hline $\begin{array}{l}\text { Valores de F } \\
\text { (concentraciones) }\end{array}$ & & $90.9 * *$ & $93.4 * *$ & $89.3 * *$ \\
\hline
\end{tabular}

Los datos son expresados como la media \pm ee de 15 larvas pesadas individualmente. Medias con letras iguales en cada columna no son estadísticamente diferentes entre sí (Kruskall-Wallis, 0.05). ** Significativo a $\mathrm{P} \leq 0.001$.

La actividad ovicida de spinosad, metoxifenocida y azadiractina varía de acuerdo con la especie del insecto tratado (Adán et al., 1996; Huang y Subramanyam, 2003; Medina et al., 2001), el solvente utilizado para aplicar el compuesto y la edad de los huevos (Pineda et al., 2004). En el presente estudio solamente spinosad y azadiractina disueltos en SDS causaron mortalidad significativa en huevos $<48$ h de edad de $S$. frugiperda. Sin embargo, en ambos insecticidas no se encontró una relación positiva entre concentración y mortalidad. En estudios previos se observó que la concentración máxima recomendada en campo de spinosad (120 mg de i.a. $\mathrm{L}^{-1}$ ) causó $49 \%$ de mortalidad en huevos de Heliothis virescens (Fabricius) (Bret et al., 1997). Pineda et al. (2004) determinaron las $\mathrm{CL}_{50}$ de este compuesto disuelto en acetona para huevos de edades menores de $24 \mathrm{~h}\left(0.20 \mathrm{mg}\right.$ de i.a. $\left.\mathrm{L}^{-1}\right)$ y de entre 24 y $48 \mathrm{~h}\left(0.15 \mathrm{mg}\right.$ de i.a. $\left.\mathrm{L}^{-1}\right)$ de Spodoptera littoralis (Boisduval).

En este caso se supone que el solvente facilitó la penetración del insecticida a través del corion delgado y liso de los huevos de este insecto, ya que los solventes orgánicos pueden facilitar la deposición y penetración de los insecticidas en la cutícula de los insectos (Adán et al., 1996). En contraste, spinosad a concentraciones altas (por ejemplo, $1000 \mathrm{mg}$ i.a. $\mathrm{L}^{-1}$ ) no causó efecto ovicida sobre la mosca de la fruta Ceratitis capitata Wiedemann (Adán et al., 1996) ni en el depredador Chrysoperla carnea (Stephens) (Medina et al., 2001), quizás debido a que el corion de los huevos de estos insectos es grueso y esponjoso, lo que evita la entrada del compuesto.

El efecto ovicida causado por azadiractina se ha estudiado ampliamente en insectos de diferentes órdenes, como en los lepidópteros $H$. virescens y Erias vitella $\mathrm{F}$. (Gahukar, 2000), los coleópteros Cosmopolites sordidus (Germar) (Musabyimana et al., 2001), Epilachna varivestis Mulsant, Leptinotarsa decemlineata (Say), y en el díptero Stomoxys calcitrans (L.) (Schmutterer, 1990). Sin embargo, este efecto puede variar con la formulación del compuesto. Al respecto, Aling $®$ causó actividad ovicida sobre $C$. capitata pero no la formulación Neem-e ${ }^{\circledR}$ (Adán et al., 1998). De igual forma, los huevos de Phthorimaea operculella (Zeller) (Iannacone y Lamas, 2003) y C. carnea (Medina et al., 2001) no fueron afectados por Neem$\mathrm{X} ®$ y Aling ${ }^{\circledR}$. En el presente estudio, azadiractina provocó una mortalidad significativa en huevos de $S$. frugiperda hasta seis veces mayor que en la del testigo. Este efecto es de importancia ya que se podría reducir el establecimiento del gusano cogollero en el cultivo de maíz y como consecuencia también el daño. Azadiractina es el principio activo más importante presente en las hojas, flores, tallos y frutos del árbol del nim, del cual en México hay plantaciones, por lo que los agricultores de bajos recursos pueden extraer este compuesto a través de métodos rústicos y utilizarlo en la protección de sus cultivos, como se hace en la India (Musabyimana et al., 2001).

Los resultados obtenidos con metoxifenocida coinciden con los de Charmillot et al. (1994), quienes reportaron que tebufenocida (el primer compuesto acelerador de la muda disponible comercialmente) no afectó a los huevos de Lobesia botrana Dennis \& Schiffermüller y Eupoecilia ambiguella Hübner. En contraste, tebufenocida y metoxifenocida disueltos en una mezcla de agua + acetona, o sólo en acetona, causaron una mortalidad significativa de huevos de Ostrinia nubilalis (Hübner) (Trisyono y Chippendale, 1997), Diatraea grandiosella Dyar (Trisyono y Chippendale, 1998), Diatraea saccharalis (Fabricius) (Rodríguez et al., 2001) y S. littoralis (Pineda et al., 2004), lo cual parece corroborar la propiedad que tiene la acetona de penetrar el corion de los huevos.

Metoxifenocida y spinosad redujeron significativamente la supervivencia de larvas que emergieron de huevos tratados. La alta mortalidad registrada en larvas recién emergidas puede deberse a que éstas se intoxicaron con los residuos de los insecticidas, tanto por ingestión al masticar parte del corion del huevo al momento de la emergencia o por contacto con el papel de estraza que 
sirvió como sustrato de oviposición. Estos resultados coinciden con los de Pineda et al. (2004), quienes reportaron que spinosad y metoxifenocida en concentraciones $\geq$ $10 \mathrm{mg}$ i.a. $\mathrm{L}^{-1}$ causaron $100 \%$ de mortalidad en larvas recién emergidas de huevos. Estos efectos también son importantes, ya que el número de individuos puede reducirse y como consecuencia la población de la plaga se mantendría por debajo del umbral económico.

En este estudio se observó que spinosad, metoxifenocida y azadiractina fueron más tóxicos por ingestión que por aplicación tópica, lo cual concuerda con la literatura disponible acerca de la actividad de estos tres insecticidas (Pineda et al., 2004, 2006; Schneider et al., 2004). En general, el orden de toxicidad, desde el menos al más tóxico, fue azadiractina < metoxifenocida < spinosad. La alta toxicidad presentada por spinosad en los ensayos por ingestión se atribuye a su modo de acción, pues es un neurotóxico, por lo que su velocidad de acción es más rápida que la de metoxifenocida y azadiractina.

Los efectos subletales observados en los insectos sobrevivientes, como reducción de peso, malformaciones y reducción de la capacidad reproductiva, tienen impacto negativo sobre su dinámica poblacional (Pineda et al., 2004). En el presente trabajo, spinosad causó una disminución significativa del peso de larvas de tercer estadio de S. frugiperda tratadas tópicamente, similar a lo observado por Yee y Toscano (1988) en larvas del tercer y quinto estadio de Spodoptera exigua (Hübner) alimentadas con hojas de lechuga (Lactuca sativa L.) tratadas con este compuesto. Este efecto podría estar relacionado con la acción neurotóxica de spinosad, que causa parálisis en insectos intoxicados (Watson, 2001) y éstos dejan de alimentarse y crecen lentamente (Pineda et al., 2006).

Metoxifenocida también redujo el peso de las larvas tratadas tópicamente, similar a lo registrado en larvas neonatas y de cuarto estadio de $S$. littoralis (Pineda et al., 2006), y en larvas de cuarto estadio de $D$. grandiosella (Trisyono y Chippendale, 1998) cuando el compuesto se suministró en la dieta. Como se ha reportado para otros compuestos aceleradores de la muda, la reducción de peso puede deberse a la actividad de metoxifenocida al ensamblar continuamente con los receptores ecdisteroidales de las larvas de lepidópteros (Smagghe et al., 2004). Además, Smagghe et al. (1997) reportaron que tebufenocida provocó alteraciones en el intestino en larvas de Chrysodeixis chalcites Esper, lo cual sugiere que tales larvas dejan de alimentarse y por ello no aumentan de peso.

La inhibición del crecimiento en larvas de Lepidoptera causada por azadiractina depende del método de aplicación, que es mayor cuando las larvas lo ingieren o cuando se suministra por inyección, que cuando se aplica tópicamente. La reducción de peso causada por este compuesto en larvas de tercer estadio de $S$. frugiperda tratadas de manera tópica fue inversamente proporcional al incremento de la dosis, similar a lo observado en larvas de Spodopera exempta (Walter) (Tanzubil y McCaffery, 1990). Este efecto se debe a la acción repelente de la azadiractina ya que actúa directamente sobre los centros que controlan la alimentación y metabolismo, lo que ocasiona que el consumo de alimento sea inversamente proporcional al incremento de la concentración (Adel y Sehnal, 2000).

\section{CONCLUSIONES}

Spinosad y azadiractina mostraron mayor efecto ovicida que metoxifenocida, por lo que los dos primeros compuestos podrían utilizarse en la etapa inicial del cultivo de maíz, de acuerdo con la presencia de los adultos de $S$. frugiperda. La alta actividad de spinosad y metoxifenocida en larvas de tercer estadio indica que pueden usarse en esta etapa de vida en los cultivos de maíz donde esta plaga causa daños importantes. Además, los insecticidas biorracionales como los aquí evaluados, son compatibles con los agentes de control biológico (parasitoides, depredadores y entomopatógenos), y permiten que éstos actúen como reguladores naturales de poblaciones plaga.

\section{AGRADECIMIENTOS}

Al Programa de Mejoramiento al Profesorado (PROMEP-PTC-101), a la Fundación Internacional para la Ciencia (IFS, por sus siglas en inglés), al Consejo Estatal de Ciencia y Tecnología (COECYT)-Michoacán y a la Coordinación de la Investigación Científica de la Universidad Michoacana de San Nicolás de Hidalgo, por el financiamiento otorgado, que incluyó becas de licenciatura para María Cristina Zamora y María Soledad Nieto de parte del COECYT-Michoacán y PROMEP, respectivamente.

\section{BIBLIOGRAFÍA}

Adán A, P Del Estal, F Budia, M González, E Viñuela (1996) Laboratory evaluation of the novel naturally derived compound spinosad against Ceratitis capitata. Pestic. Sci. 48:261-268.

Adán A, J Soria, P Del Estal, C Sánchez-Brunete, E Viñuela (1998) Acción diferencial de dos formulaciones de azadiractina sobre los estados de desarrollo de Ceratitis capitata. Bol. San.Veg. Plagas 24:1009-1018.

Adel M M, F Sehnal (2000) Azadirachtin potentiates the action of ecdysteroid agonist RH-2485 in Spodoptera littoralis. J. Insect Physiol. 46:267-274.

Bret L, L Larson, R Scroonover, C Sparks, D Thompson (1997) Biological properties of spinosad. Down to Earth 52:6-13.

Charmillot J, R Favre, D Pasqueir, M Rhyn, A Scalco (1994) Effect du régulater de croissance d'insectes ( $\mathrm{RCI})$ tébufenozide surles oeufs, les larves et les papillons des vers de la grappe 
Lobesia botrana Den \& Schiff. et Eupoecilia ambiguella $\mathrm{Hb}$ Bull. Soc. Entomol. Suisse 67:393-402.

Dhadialla S, R Carlson, P Le (1998) New insecticides with ecdysteroidal and juvenile hormone activity. Annu. Rev. Entomol. 43:545-569.

Gahukar R T (2000) Use of neem products/pesticides in cotton pest management. Int. J. Pest Manage. 46:149-160.

Huang F, B Subramanyam (2003) Response of Corcyra cephalonica (Stainton) to pirimiphos-methyl, spinosad, and combinations of pirimiphos-methyl and synergized pyrethrins. Pest Manage. Sci. 60:191-198.

Hruska A J, F Gould (1997) Fall armyworm (Lepidoptera: Noctuidae) and level and temporal occurrence on maize yield in Nicaragua. J. Econ. Entomol. 90:611-622.

Iannacone J, G Lamas (2003) Efecto insecticida de cuatro extractos botánicos y del cartap sobre la polilla de la papa Phthorimaea operculella (Zeller) (Lepidoptera: Gelechiidae), en Perú. Entomotropica 18:95-105.

Martínez A M, P Caballero, M Villanueva, N Miralles, I San Martín, E López, T Williams (2004) Formulation with an optical brightener does not increase probability of developing resistance to Spodoptera frugiperda nucleopolyedrovirus in the laboratory. J. Econ. Entomol. 97:1202-1208.

Medina M P, F Budia, L Tirry, G Smagghe, E Viñuela (2001) Compatibility of spinosad, tebufenozide and azadirachtin with eggs and pupae of the predator Chrysoperla carnea (Stephens) under laboratory conditions. Biocontrol Sci. Technol. 11:597610

Morillo F, A Notz (2003) Resistencia de Spodoptera frugiperda (Smith) (Lepidoptera: Noctuidae) a lambdacihalotrina y metomil. Entomotropica 16:79-87.

Musabyimana T, R C Sexeana, E W Kairu, C P K O Ogol, R Khan (2001) Effects of neem seed derivates on behavioural and physiological responses of the Cosmopolites sordidus (Coleoptera: Curculionidae). J. Econ. Entomol. 94: 449-454.

Pineda S, F Budia, M I Schneider, A Gobbi, E Viñuela, J Valle, P Del Estal (2004) Effect of two biorational insecticides, spinosad and methoxyfenozide, on Spodoptera littoralis (Lepidoptera: Noctuidae) under laboratory conditions. J. Econ. Entomol. 97:1906-1911.

Pineda S, G Smagghe, M I Schneider, P Del Estal, E Viñuela, A M Martínez, F Budia (2006) Toxicity and pharmacokinetics of spinosad and methoxyfenozide to Spodoptera littoralis (Lepidoptera: Noctuidae). Environ. Entomol. 35:856-864.

Poitout S, R Bues (1974) Elevage de chenilles de veingt-huit espeses de lépidopterés Noctuidae. Ann. Zool. Ecol. Anim. 6: 341-411.

Rodríguez M, A Otea, E Reagan (2001) Selection, egg viability, and fecundity of the sugarcane borer (Lepidoptera: Crambidae) with tebufenozide. J. Econ. Entomol. 94:1553-1557.

Schmutterer H (1990) Properties of natural pesticides from the neem tree, Azadirachta indica. Annu. Rev. Entomol. 35:271-297.

Schneider M, G Smagghe, S Pineda, E Viñuela (2004) Action of insect growth regulator insecticides and spinosad on life history parameters and absorption in third instar larvae of the endoparasitoid Hyposoter didymator. Biol. Control 31:189. 198.

Smagghe G, E Viñuela, F Budia, D Degheele (1997) Effects of the non-steroidal ecdysteroid mimic tebufenozide on tomato looper Chrysodeixis chalcites (Lepidoptera: Noctuidae). An ultrastructural analysis. Arch. Insect Biochem. Physiol. 35:179-190.

Smagghe G, D Bylemans, P Medina, F Budia, J Avilla, E Viñuela (2004) Tebufenozide distorted codling moth larval growth and reproduction, and controlled field populations. Ann. Appl. Biol. 145:291-298.

Tanzubil B P, R A McCaffery (1990) Efects of azadirachtin on reproduction in the African armyworm (Spodoptera exempta). Entomol. Exp. Appl. 57:115-121.

Thompson G D, R Dutton, T C Sparks (2000) Spinosad-a case study: an example from a natural products discovery programme. Pest Manage. Sci. 56:696-702.

Trisyono A, M Chippendale (1997) Effect of the nonsteroidal ecdysone agonists, methoxyfenozide, and tebufenozide, on the European corn borer (Lepidoptera: Pyralidae). J. Econ. Entomol. 90:1486-1492.

Trisyono A, M Chippendale (1998) Effect of the ecdysone agonists, RH-2485 and tebufenozide, on the southwestern corn borer, Diatraea grandiosella. Pestic. Sci. 53:177-185.

Watson G B (2001) Actions of insecticidal spinosyns on $\gamma$-aminobutyric acid receptors from small-diameter cockroach neurones. Pestic. Biochem. Physiol. 71:20-28.

Yee W L, N C Toscano (1998) Laboratory evaluations of synthetic and natural insecticides on beet armyworm (Lepidoptera: Noctuidae) damage and survival on lettuce. J. Econ. Entomol. 91:56-63. 